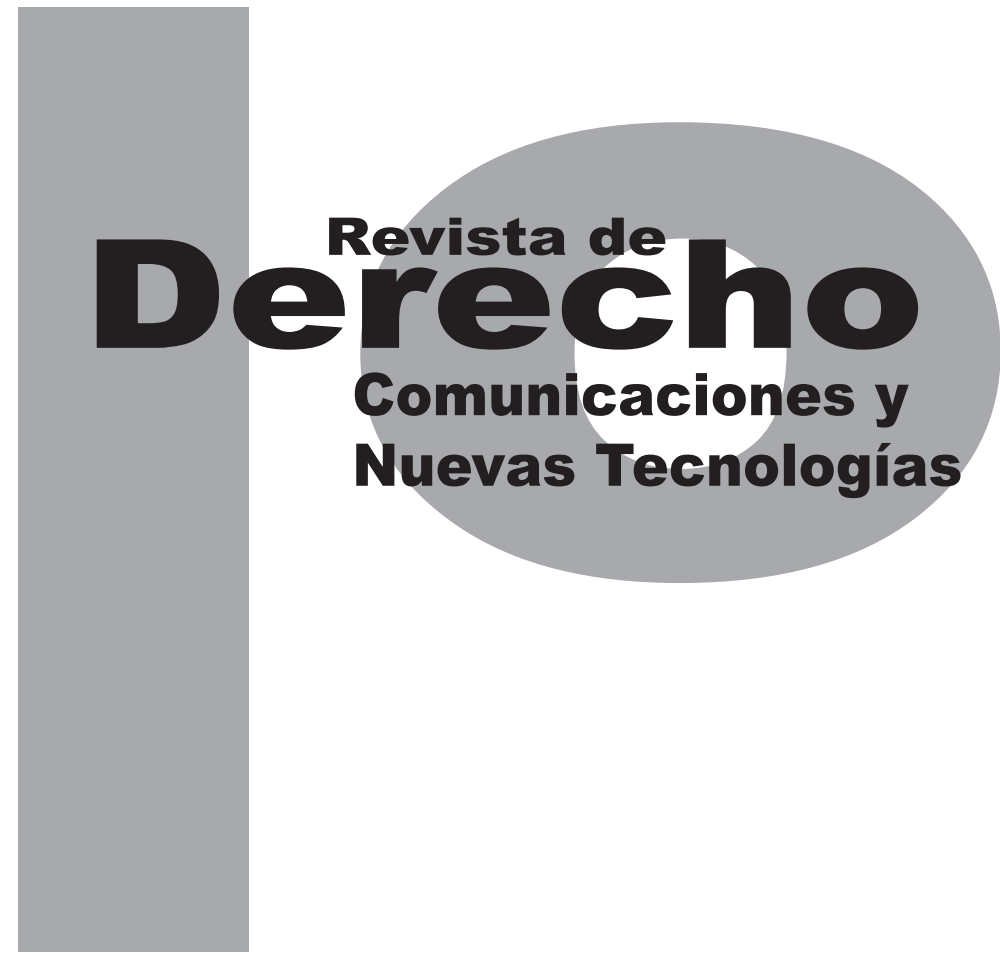

\title{
MARCO JURÍDICO-ADMINISTRATIVO DEL GOBIERNO DIGITAL EN COSTA RICA
}

\author{
M.P.A. Jennifer ISABEl ARROYo ChACón
}

Universidad de los Andes

Facultad de Derecho

Revista de Derecho, Comunicaciones y Nuevas Tecnologías

No. 10, Julio - Diciembre de 2013. ISSN 1909-7786 


\title{
Marco jurídico-administrativo del gobierno digital en Costa Rica*
}

\author{
M.P.A. Jennifer Isabel Arroyo Chacón ${ }^{* *}$
}

\section{RESUMEN}

El Gobierno Digital se refiere al uso de las Tecnologías para facilitar la interacción entre el Estado y la ciudadanía. Costa Rica ha ratificado importantes instrumentos internacionales sobre el tema y ha designado esta función a distintos actores dentro de la estructura de la administración pública. No obstante, esa diversidad de actores ha generado problemas de coordinación, comunicación, políticas carentes de permanencia en el tiempo, escases de recursos económicos; lo cual, aunado al hecho de que no siempre las instituciones cumplen con sus funciones adecuadamente y que se carece de
E-government is the use of technology to facilitate interaction between the government and citizens. Costa Rica has ratified major international instruments on the subject and designates its promotion in various actors within the public administration structure. However, this diversity of actors have led problems of coordination, communication, lacking political permanence in time and shortage of recourses, which together with the fact those institutions do not always fulfill their functions properly, and that there are not a real leadership on the issue. These facts have prevented the E-government reaches the

Cómo citar este artículo: Arroyo Chacón, J. (Diciembre, 2013). Marco jurídico-administrativo del Gobierno Digital en Costa Rica. Revista de Derecho, Comunicaciones y Nuevas Tecnologías, 10.

** Es Abogada, Contadora Pública Autorizada (auditora) C.P.A. y Administradora Pública incorporada al Colegio de Abogados, Colegio de Contadores Públicos de Costa Rica y al Instituto de Auditores Internos de Costa Rica. Certificada en Normas Internacionales de Auditoría (NIAS) por el Colegio de Contadores Públicos de Costa Rica. Posee un Diplóme d'supérieures spécialisées en Administration Publique de la Universidad de Quebec, Canadá y una Maestría en Administración Pública con énfasis en Gestión Pública de la Universidad de Costa Rica. Labora en la Contraloría General de la República y ha fungido como profesora de los cursos de derecho comercial, laboral, tributario y administración pública para los estudiantes de las carreras de administración de negocios y contaduría pública en distintas universidades públicas y privadas. Ha publicado libros y artículos vinculados con las áreas del derecho, la contaduría, la auditoría y la administración pública en revistas nacionales e internacionales. Correo: prof.jenniferarroyo@yahoo. com, http://www.linkedin.com/pub/jennifer-isabel-arroyo-chac\%C3\%B3n/2b/ba7/217 
un verdadero liderazgo en el tema, ha impedido que el Gobierno Digital alcance los niveles de desarrollo deseados; elementos que se analizan y desarrollan en el presente documento.

Palabras Clave: Gobierno Digital, Normativa Internacional en Gobierno Digital, Administración Pública Costarricense desired developments levels. This document discusses and develops these elements.

KeYWORDS: E-Government, International Law in E-government, Costa Rican Public Administration. 


\section{SUMARIO}

Introducción - I. MARCO CONCEPTUAL - A. Tecnologías de la Información y la Comunicación - B. Sociedad de la Información - C. Gobierno Digital - II. MARCO JURÍDICO DEL GOBIERNO DIGITAL EN COSTA RICA - A. Convenios internacionales - 1. Carta Iberoamericana de Gobierno Electrónico -2. Carta Iberoamericana de calidad en la Gestión Pública - 3. Segunda conferencia ministerial sobre la sociedad de la información en América Latina y el Caribe - B. Normativa nacional -III. PRINCIPALES ACTORES DEL GOBIERNO DIGITAL EN COSTA RICA - A. Secretaría Técnica del Gobierno Digital - B. Comisión Intersectorial de Gobierno Digital - C. Ministerio de Ciencia y Tecnología -D. Comisión de Tecnologías de la Información y la Comunicación (CONATIC) - IV. PRINCIPALES OBSTÁCULOS PARA EL AVANCE DEL GOBIERNO DIGITAL EN COSTA RICA - V. Propuesta para superar los obstáculos al avance del Gobierno Digital en Costa Rica - VI. CONCLUSIONES - Bibliografía 
Introducción

Las Tecnologías de la Información y la Comunicación han cambiado la manera en la que se desarrolla la sociedad y el sector público no ha escapado a dichos cambios, por lo que se han introducido nuevas formas de prestar servicios públicos utilizando los beneficios que brindan estas tecnologías en procura de alcanzar servicios eficientes, oportunos y a menores costos.

Precisamente, el Gobierno Digital surge como respuesta a este cambio social y a la demanda de los ciudadanos de contar con servicios públicos eficientes, expeditos, oportunos, gratuitos y disponibles las 24 horas del día, los 7 días de la semana.

De esta manera, en los últimos años se ha promocionado la iniciativa del Gobierno Digital en América Latina como un medio para satisfacer las necesidades del ciudadano por medio de las Tecnologías de la Información y la Comunicación y, a su vez, cumplir con principios de transparencia y rendición de cuentas, lo que ha generado la aprobación por parte de los gobiernos de documentos en donde se comprometen a prestar estos servicios a sus ciudadanos.

Dentro de estos gobiernos, que se han comprometido con el desarrollo del Gobierno Digital, se incluye Costa Rica, que ha ratificado convenios internacionales aprobando normativa interna y designado esta tarea a diferentes actores dentro del organigrama público.
No obstante, el avance logrado por este país en la materia no posee el nivel de desarrollo deseado, por lo que conviene analizar el marco jurídico que regula la materia en el país a fin de determinar las posibles causas que han detenido el avance de este proyecto.

Por lo tanto, el objetivo del presente documento es describir el marco jurídico aprobado por Costa Rica, así como los distintos actores que a nivel administrativo son responsables de impulsar la implementación y desarrollo del Gobierno Digital en el país, con el fin de llegar a deducir algunas de las causas que han impedido que este proyecto avance al ritmo deseado y proponer recomendaciones al respecto.

\section{MARCO CONCEPTUAL}

\section{A. Tecnologías de la Información y la Comunicación}

La Sociedad de la Información y el Gobierno Digital se basan en las Tecnologías de la Información y la Comunicación y poseen su origen, precisamente, en la revolución que dichas tecnologías han introducido en la vida en sociedad, de tal manera que antes de entrar a analizar el tema objeto del presente documento, conviene repasar el término: Tecnologías de la Información y la Comunicación, el cual fue definido por el CEPAL en 2003, de la siguiente manera.

Las TIC se definen como sistemas tecnológicos mediante los que se recibe, manipula y procesa información, y que facilitan la comunicación entre dos o más interlocutores. Por lo 
tanto, las TIC son algo más que informática y computadoras, puesto que no funcionan como sistemas aislados, sino en conexión con otras mediante una red. También son algo más que tecnologías de emisión y difusión (como televisión y radio), puesto que no sólo dan cuenta de la divulgación de la información, sino que además permiten una comunicación interactiva. El actual proceso de "convergencia de TIC" (es decir, la fusión de las tecnologías de información y divulgación, las tecnologías de la comunicación y las soluciones informáticas) tiende a la coalescencia de tres caminos tecnológicos separados en un único sistema que, de forma simplificada, se denomina TIC (o la "red de redes"). (CEPAL, Katz, y Hilbert, 2003, Los caminos hacia una Sociedad de la Información en América Latina y el Caribe, p. 3).

Como puede verse, este concepto resulta más amplio que simplemente hardware o software porque se vincula con la función que deben tener las Tecnologías de la Información y la Comunicación, que es permitir la comunicación y divulgación de la información, lo cual ha revolucionado nuestro modo de vida y de tal revolución no ha escapado el Estado y la administración pública.

\section{B. Sociedad de la Información}

La introducción de las Tecnologías de la Información y la Comunicación ha provocado profundos cambios en nuestras vidas; y por ende, en el modelo de sociedad en el cual vivimos, tan profundas que actualmente se nos Ilama: la Sociedad de la Información.

Pero, ¿qué es lo que realmente significa e implica el término Sociedad de la Información? En este punto se debe señalar que se han realizado varios esfuerzos por definir este término, entre ellos los siguientes:

El concepto de 'sociedad de la información' hace referencia a un paradigma que está produciendo profundos cambios en nuestro mundo al comienzo de este nuevo milenio. Esta transformación está impulsada principalmente por los nuevos medios disponibles para crear y divulgar información mediante tecnologías digitales. Los flujos de información, las comunicaciones y los mecanismos de coordinación se están digitalizando en muchos sectores de la sociedad, proceso que se traduce en la aparición progresiva de nuevas formas de organización social y productiva. (CEPAL, Katz, y Hilbert, 2003, p. 1).

La sociedad de la información es aquella sociedad que ordena, estructura su funcionamiento (modo de vida, forma de relaciones, modo de trabajo, etc.) en torno a las tecnologías de la información y la comunicación y convierte a la información en un factor de producción, intercambio y conocimiento. (Fernando, 2004, Los Sistemas de Información en la Sociedad del Conocimiento, p. 4).

El concepto de "sociedad de la información" es muy complejo y su nivel de desarrollo es aún incipiente. La comunidad intelectual tendrá que reducir esta complejidad mediante un proceso de abstracción que permita expresar la "realidad" paradigmática en términos de entidades concretas e interrelaciones. Será necesario establecer los términos y los esquemas que permitan debatir el concepto de "sociedad de la información", puesto que este modelo es esencial para plantear objetivos específicos de desarrollo. El marco conceptual utilizado por la 
CEPAL se basa en las características generales de las tecnologías de la información y las comunicaciones (TIC) y del proceso de digitalización resultante, que son el núcleo de este paradigma emergente. (CEPAL, Katz, y Hilbert, 2003, Los caminos hacia una Sociedad de la Información en América Latina y el Caribe, p. 11).

Como puede notarse, estas definiciones se refieren a la sociedad de la información a partir de los cambios que se han implementado en la vida en sociedad con la introducción de estas tecnologías. En este punto, se hace obligatoria la referencia a la Declaración de Principios de la Cumbre Mundial de Sociedad de la Información, en donde las naciones, ponen al ser humano como el centro de la sociedad de la información, por encima de las TIC's, de la siguiente manera:

1. Nosotros, los representantes de los pueblos del mundo, reunidos en Ginebra del 10 al 12 de diciembre de 2003 con motivo de la primera fase de la Cumbre Mundial sobre la Sociedad de la Información, declaramos nuestro deseo y compromiso comunes de construir una Sociedad de la Información centrada en la persona, integradora y orientada al desarrollo, en que todos puedan crear, consultar, utilizar y compartir la información y el conocimiento, para que las personas, las comunidades y los pueblos puedan emplear plenamente sus posibilidades en la promoción de su desarrollo sostenible y en la mejora de su calidad de vida, sobre la base de los propósitos y principios de la Carta de las Naciones Unidas y respetando plenamente y defendiendo la Declaración Universal de Derechos Humanos. (Organización de las Naciones Unidas, y Unión Internacional de Telecomunicaciones, 2003, Declaración de
Principios de la Cumbre Mundial de Sociedad de la Información, Num. 1, párr. 1).

La Cumbre Mundial de la Sociedad de la Información centra su definición de sociedad de la información, no en las Tecnologías de la Información y la Comunicación, sino en el ser humano, lo cual posee un gran impacto pues es responsabilidad de los gobiernos tratar el tema de las tecnologías, siempre en función del beneficio que éstas le traen al ser humano. Este reconocimiento del ser humano como centro de la Sociedad de la Información ha impactado el esquema jurídico vigente e incluso ha sido reconocido por la propia Sala Constitucional en el voto 12790-2010, de la siguiente manera:

En cuanto a este último punto, debe decirse que el avance en los últimos veinte años en materia de tecnologías de la información y comunicación (TIC's) ha revolucionado el entorno social del ser humano. Sin temor a equívocos, puede afirmarse que estas tecnologías han impactado el modo en que el ser humano se comunica, facilitando la conexión entre personas e instituciones a nivel mundial y eliminando las barreras de espacio y tiempo. En este momento, el acceso a estas tecnologías se convierte en un instrumento básico para facilitar el ejercicio de derechos fundamentales como la participación democrática (democracia electrónica) y el control ciudadano, la educación, la libertad de expresión y pensamiento, el acceso a la información y los servicios públicos en línea, el derecho a relacionarse con los poderes públicos por medios electrónicos y la transparencia administrativa, entre otros. Incluso, se ha afirmado el carácter de derecho fundamental que reviste el acceso a estas tec- 
nologías, concretamente, el derecho de acceso a la Internet o red de redes. (2010, Acceso a Internet como Derecho Fundamental, Título V, párr. 1).

\section{Gobierno Digital}

La revolución que estamos viviendo ha demandado cambios de parte del Estado y la administración pública, y ha obligado a que éste modifique la manera tradicional de ejercer su función y se apoye en las Tecnologías de la Información y la Comunicación para prestar mejores servicios a la Sociedad de la Información que se lo exige.

El tema de Gobierno Digital resulta tan importante que diversos organismos internacionales se han dado la tarea de definir este término de la siguiente manera:

Se refiere al uso de tecnologías de información por parte de las agencias gubernamentales como por ejemplo, las redes de área extendida, Internet y la informática móvil. Estas tecnologías poseen el potencial para transformar las relaciones con los ciudadanos, empresas, y otras ramas de gobierno. Estas tecnologías pueden tener diversos fines: mejorar la calidad de los servicios gubernamentales a los ciudadanos, promover las interacciones con las empresas e industrias, fortalecer la participación ciudadana a través del acceso a la información y más eficiente administración gubernamental. Los beneficios esperados son: disminuir la corupción, promover la transparencia y convivencia y reducir costos. (Banco Mundial, s.f., Concepto de Gobierno Digital, párr. 1).
A los efectos de la presente Carta Iberoamericana se entienden las expresiones de "Gobierno Electrónico" y de "Administración Electrónica" como sinónimas, ambas consideradas como el uso de las TIC en los órganos de la Administración para mejorar la información y los servicios ofrecidos a los ciudadanos, orientar la eficacia y eficiencia de la gestión pública e incrementar sustantivamente la transparencia del sector público y la participación de los ciudadanos. Todo ello, sin perjuicio de las denominaciones establecidas en las legislaciones nacionales. (XVII Cumbre Iberoamericana de Jefes de Estado y de Gobierno, 2007, Carta Iberoaméricana de Gobierno Electrónico, Punto 3, párr. 1).

Uso de las Tecnologías de Información y Comunicación por parte de las instituciones de gobierno, para mejorar cualitativamente los servicios e información ofrecidos a los ciudadanos, aumentar la eficiencia y eficacia de la gestión pública e incrementar sustantivamente la transparencia del sector público y la participación ciudadana. (Organización de Estados Americanos, 2011, Módulo I: Introducción al Gobierno Electrónico, p. 7).

Como puede apreciarse, el Gobierno Digital es el concepto que define la introducción de las Tecnologías de la Información y la Comunicación en la administración pública como un medio para mejorar la eficiencia, eficacia y transparencia en la función pública.

Por su parte, la Secretaría Técnica de Gobierno Digital de Costa Rica ha definido este concepto de la siguiente manera: 
El Gobierno Digital se refiere al uso creativo de las tecnologías de información para transformar la manera como interactúa el Gobierno con las empresas y los ciudadanos. Es una forma de modernizar al Estado, simplificando y haciendo más eficiente la prestación de servicios y la realización de trámites en la administración pública. (Secretaría Técnica de Gobierno Digital de Costa Rica, s.f., ¿Qué es Gobierno Digital, párr. 1).

En este punto se considera relevante señalar que Gobierno Digital no es sólo poner computadoras en las oficinas públicas o que las instituciones tengan página web, sino que implica un cambio de paradigma, un replanteo de gestiones y procesos, de tal manera que las instituciones se propongan mejorar el servicio y cambiar su metodología de trabajo para satisfacer de mejor manera las necesidades del ciudadano.

\section{MARCO JURÍDICO DEL GOBIERNO DIGITAL EN COSTA RICA}

\section{A. Convenios internacionales}

Costa Rica ha firmado convenios internacionales relevantes en el tema de Gobierno Digital, constituyéndose éstos en la base para regular el Gobierno Digital en el país.
Los citados convenios jurídicos son:

Tabla 1. Fuente: creación propia con datos oficiales

\section{Convenios Internacionales}

\begin{tabular}{|l|l|l|}
\hline \multicolumn{3}{|c|}{ Convenios Internacionales } \\
\hline Instrumento & Aprobación & Año \\
\hline $\begin{array}{l}\text { Carta iberoameri- } \\
\text { cana de gobierno } \\
\text { electrónico }\end{array}$ & $\begin{array}{l}\text { Aprobada por la IX Con- } \\
\text { ferencia Iberoamericana } \\
\text { de Ministros de Adminis- } \\
\text { tración Pública y Refor- } \\
\text { ma del Estado }\end{array}$ & 2007 \\
\hline $\begin{array}{l}\text { Carta iberoamerica- } \\
\text { na de calidad en la } \\
\text { gestión pública }\end{array}$ & $\begin{array}{l}\text { Aprobada por la X Con- } \\
\text { ferencia Iberoamericana } \\
\text { de Ministros de Adminis- } \\
\text { tración Pública y Refor- } \\
\text { ma del Estado }\end{array}$ & 2008 \\
\hline $\begin{array}{l}\text { Segunda conferen- } \\
\text { cia ministerial sobre } \\
\text { la sociedad de la } \\
\text { información en } \\
\text { américa latina y el } \\
\text { caribe }\end{array}$ & $\begin{array}{l}\text { Plan de Acción sobre la } \\
\text { Sociedad de la Informa- } \\
\text { ción de América Latina y } \\
\text { el Caribe eLAC 2010 }\end{array}$ & 2010 \\
\hline
\end{tabular}

Los anteriores documentos poseen gran relevancia ya que constituyen el compromiso de los gobiernos en la implementación del Gobierno Digital en sus países y, para ello, se requiere de un marco jurídico que lo promueva y de las gestiones institucionales y administrativas necesarias para su implementación, por lo que conviene mencionarlos brevemente.

\section{Carta Iberoamericana de Gobierno Electrónico}

La Carta Iberoamericana de Gobierno Electrónico nace un como el esfuerzo de los países iberoamericanos por reducir la brecha digital que existe en la región, de comprometer a los gobiernos de la región a realizar gestiones tendientes a implementar el Gobierno Digital y democratizar el acceso de las Tecnologías de la Información y la Comunicación para todos los países. 
Dicho instrumento posee dos objetivos claramente definidos, que son:

- Un objetivo final y directo: reconocer a los ciudadanos un derecho que les facilite su participación en la gestión pública y sus relaciones con las Administraciones Públicas y que contribuya también a hacer éstas más transparentes y respetuosas con el principio de igualdad, a la vez que más eficaces y eficientes.

- Un objetivo estratégico e indirecto: promover la construcción de una sociedad de información y conocimiento, inclusiva, centrada en las personas y orientada al desarrollo. (XVII Cumbre Iberoamericana de Jefes de Estado y de Gobierno, 2007, p. 5).

Un primer objetivo consiste en reconocer el derecho del ciudadano a recibir información transparente, de manera eficaz y eficiente de parte del Estado, lo cual va a permitir alcanzar el segundo objetivo, que se refiere a la construcción de una sociedad de la información inclusiva y centrada en las personas, aspectos que se alcanzan con la adecuada implementación del Gobierno Digital.

Para lograr estos objetivos, los países deben ajustar su normativa interna y crear las instancias administrativas responsables de ejecutar proyectos en este sentido, así quedó claramente establecido en el punto 7 de dicha carta, que a la letra dice:

La implantación del Gobierno Electrónico comporta el reconocimiento por parte de los Estados Iberoamericanos del derecho de los ciudadanos a relacionarse electrónicamente con sus
Gobiernos y Administraciones Públicas. Lo que supone que las Administraciones estén interrelacionadas entre sí a fin de simplificar los procedimientos. Las leyes de acceso a la información pública establecidas en algunos países de la región apuntan en esa dirección. (XVII Cumbre Iberoamericana de Jefes de Estado y de Gobierno, 2007, p. 10).

Esta carta instaura una serie de principios bajo los cuales se fundamenta el Gobierno Digital y para efectos de los intereses del presente documento, se destaca el principio de legalidad, entendido como:

Principio de legalidad: de forma que las garantías previstas en los modos tradicionales de relación del ciudadano con el Gobierno y la Administración se mantengan idénticas en los medios electrónicos. Los trámites procedimentales, sin perjuicio de su simplificación general, constituyen para todos los ciudadanos garantías imprescindibles. El principio de legalidad también comprende el respeto a la privacidad, por lo que el uso de comunicaciones electrónicas comporta la sujeción de todas las Administraciones Públicas a la observancia de las normas en materia de protección de datos personales. (XVII Cumbre Iberoamericana de Jefes de Estado y de Gobierno, 2007, p. 8).

Desde el punto de vista de la labor normativa, este principio presenta un gran reto para los países, pues les impone la obligación de, no sólo actualizar su normativa interna con el fin de impulsar la implementación del gobierno electrónico, sino que en el proceso se debe garantizar todos los derechos de los ciudadanos y la protección que recibían con los medios tradicio- 
nales; es decir, que el Gobierno Digital permita prestarle al ciudadano un servicio más eficiente y transparente, pero con las mayores garantías y condiciones de seguridad posibles.

\section{Carta Iberoamericana de calidad en la Gestión Pública}

La Carta Iberoamericana de Calidad en la Gestión Pública regula el derecho que posee el ciudadano de recibir una gestión pública de calidad, bajo parámetros de eficacia, eficiencia y buen servicio, vinculado con dos propósitos fundamentales, que son:

1. toda gestión pública debe estar referenciada a la satisfacción del ciudadano, ya sea como usuario o beneficiario de servicios y programas públicos, o como legítimo participante en el proceso formulación, ejecución y control de las políticas públicas bajo el principio de corresponsabilidad social; 2. la gestión pública tiene que orientarse para resultados, por lo que debe sujetarse a diversos controles sobre sus acciones, suponiendo entre otras modalidades la responsabilización del ejercicio de la autoridad pública por medio del control social y rendición periódica de cuentas. (XVIII Cumbre Iberoamericana de Jefes de Estado y de Gobierno, 2008, Carta Iberoamericana de Calidad en la Gestión Pública, p. 5).

Dentro de los elementos necesarios para garantizar un servicio público de calidad al ciudadano, esta carta incluye el tema del gobierno electrónico de la siguiente manera:

De conformidad con la "Carta Iberoamericana de Gobierno Electrónico”, la Administración
Pública buscará la optimización de las tecnologías de la información y la comunicación, para la mejora y acercamiento del servicio a las necesidades ciudadanas, así como de la propia gestión pública, mejora de la atención, trámites en línea, participación y transparencia. Para ello, tratará de facilitar múltiples modalidades de acceso y realización de trámites, así como el intercambio documental y de información en ambos sentidos Administración-ciudadano.

En particular, se propiciará la interconexión entre las diferentes Administraciones orientada a la simplificación de trámites para el ciudadano. (XVIII Cumbre Iberoamericana de Jefes de Estado y de Gobierno, 2008, Punto 51, párr. 1).

Lo anterior demuestra la importancia que posee el Gobierno Digital como un medio para alcanzar un servicio público eficiente y de calidad al ciudadano y que permita la comunicación entre Estado-Ciudadano, con fines de transparencia, rendición de cuentas y mejoras en la prestación del servicio.

\section{Segunda conferencia ministerial sobre la sociedad de la información en América Latina y el Caribe}

El objetivo perseguido por la Segunda conferencia ministerial sobre la sociedad de la información en América Latina y el Caribe radicaba en ratificar el compromiso de los gobiernos de fortalecer el Gobierno Digital y en que la Sociedad de la Información tuviera como centro de su desarrollo al ser humano, aspecto señalado por dicho documento de la siguiente manera: 
Nuestro deseo y determinación comunes de construir una sociedad de la información integradora, orientada al desarrollo y centrada en la persona, basada en los principios de paz, derechos humanos, solidaridad, libertad, democracia, desarrollo sostenible y cooperación, para que los seres humanos, las comunidades y los pueblos de América Latina y el Caribe puedan aprovechar plenamente sus potencialidades en la promoción de su desarrollo.

Nuestro compromiso, como gobiernos, de facilitar la inclusión de todas las partes interesadas -el sector privado, la sociedad civil, la comunidad científica y académica y cualquier otra- en el proceso de creación de una sociedad de la información basada en conocimientos compartidos, de acuerdo con los mecanismos establecidos para estos fines por la Cumbre Mundial sobre la Sociedad de la Información. (Gobierno de El Salvador, Y CEPAL, 2008, p. 1-2).

Bajo esta perspectiva se incluyen una serie de apartados vinculados con las Tecnologías de la Información y la Comunicación en los diferentes ámbitos de los servicios públicos y, en el tema de Gestión Pública, se buscar promover el uso de plataformas tecnológicas, intercambios y demás medios que permitan prestar un mejor servicio público como se cita a continuación:

Fortalecer medios de intercambio sobre servicios de gobierno electrónico, desarrollando cooperación regional para el intercambio o transferencia de tecnologías, plataformas, aplicaciones y programas informáticos, así como sus correspondientes conocimientos, habilidades y mejores prácticas. Utilizar dichas redes para la implementación de estándares de interoperabilidad de servicios gubernamenta- les electrónicos. (Gobierno de El Salvador, Y CEPAL, 2008, p. 9).

\section{B. Normativa nacional}

Con el fin de ajustar nuestra normativa interna a los mandatos de los instrumentos jurídicos ratificados por el país, se han aprobado una serie de leyes que regulan aspectos como la promoción del desarrollo tecnológico del país, la protección al ciudadano de exceso de trámites y firma digital; así como una serie de decretos específicos que persiguen los mismos fines.

Las leyes y decretos que actualmente se encuentran vigentes son:

Tabla 2. Fuente: creación propia con datos oficiales

Normativa nacional.

\begin{tabular}{|c|c|c|}
\hline $\begin{array}{l}\text { Número de } \\
\text { Instrumento }\end{array}$ & Nombre & $\begin{array}{l}\text { Fecha de } \\
\text { Aproba- }\end{array}$ \\
\hline \multicolumn{3}{|l|}{ Leyes } \\
\hline Ley №7169 & $\begin{array}{l}\text { Ley de Promoción del De- } \\
\text { sarrollo Científico y Tec- } \\
\text { nológico }\end{array}$ & $\begin{array}{l}26 \text { de junio } \\
\text { de } 1990\end{array}$ \\
\hline Ley Nº220 & $\begin{array}{l}\text { Ley Protección al Ciuda- } \\
\text { dano del Exceso de Re- } \\
\text { quisitos y Trámites Admi- } \\
\text { nistrativos. }\end{array}$ & $\begin{array}{l}4 \text { de marzo } \\
\text { del } 2002\end{array}$ \\
\hline Ley Nº 8454 & $\begin{array}{l}\text { Ley de Certificados, Fir- } \\
\text { mas Digitales y Docu- } \\
\text { mentos Electrónicos }\end{array}$ & $\begin{array}{l}30 \text { de agos- } \\
\text { to de } 2005\end{array}$ \\
\hline Ley Nº 9046 & $\begin{array}{l}\text { Ley de Traslado del Sector } \\
\text { Telecomunicaciones del } \\
\text { Ministerio de Ambiente, } \\
\text { Energía y Telecomunica- } \\
\text { ciones al Ministerio de } \\
\text { Ciencia y Tecnología }\end{array}$ & $\begin{array}{l}25 \text { de julio } \\
\text { del } 2012\end{array}$ \\
\hline
\end{tabular}




\begin{tabular}{|c|c|c|}
\hline $\begin{array}{l}\text { Decreto Ejecu- } \\
\text { tivo } N^{\circ} 31681\end{array}$ & $\begin{array}{l}\text { Crea la Comisión Nacio- } \\
\text { nal de Tecnologías de la } \\
\text { Información y la Comu- } \\
\text { nicación (CONATIC) }\end{array}$ & $\begin{array}{l}20 \text { de ene- } \\
\text { ro del } 2004\end{array}$ \\
\hline $\begin{array}{l}\text { Decreto Ejecu- } \\
\text { tivo N³5139 }\end{array}$ & $\begin{array}{l}\text { Crea Comisión Inter- } \\
\text { sectorial de Gobierno } \\
\text { Digital }\end{array}$ & $\begin{array}{l}6 \text { de abril } \\
\text { del } 2009\end{array}$ \\
\hline $\begin{array}{l}\text { Decreto Ejecu- } \\
\text { tivo } N^{\circ} 34704\end{array}$ & $\begin{array}{l}\text { Promoción del Teletra- } \\
\text { bajo en las Institucio- } \\
\text { nes Públicas }\end{array}$ & $\begin{array}{l}31 \text { de julio } \\
\text { del } 2009\end{array}$ \\
\hline $\begin{array}{l}\text { Decreto Ejecu- } \\
\text { tivo № } 35776 \\
\text {-PLAN-G-J }\end{array}$ & $\begin{array}{l}\text { Promoción del Modelo } \\
\text { de Interoperabilidad en } \\
\text { el Sector Público }\end{array}$ & $\begin{array}{l}29 \text { de ene- } \\
\text { ro del } 2010\end{array}$ \\
\hline $\begin{array}{l}\text { Decreto Ejecu- } \\
\text { tivo } N^{\circ} 35776\end{array}$ & $\begin{array}{l}\text { Promoción del Modelo } \\
\text { de Interoperabilidad en } \\
\text { el sector Público }\end{array}$ & $\begin{array}{l}1 \text { de marzo } \\
\text { del } 2010\end{array}$ \\
\hline $\begin{array}{l}\text { Decreto } \\
\text { Ejecutivo } \\
36176-\mathrm{MO}- \\
\text { PLAN }\end{array}$ & $\begin{array}{l}\text { Reforma del artículo } 1 \text { o } \\
\text { del Decreto Ejecutivo } \\
N^{\circ} \quad 35139-M P-M I D E- \\
\text { PLAN que crea la Co- } \\
\text { misión Intersectorial de } \\
\text { Gobierno Digital }\end{array}$ & $\begin{array}{l}4 \text { de oc- } \\
\text { tubre del } \\
2010\end{array}$ \\
\hline
\end{tabular}

Ahora bien, Costa Rica aún posee una deuda significativa en la materia, no sólo de lograr una verdadera implementación de las normas antes citadas, especialmente con el tema de simplificación de trámites y exceso de requisitos para trámites públicos, sino que también está pendiente la creación de una Ley de acceso a la información pública que permita definir claramente qué información puede ser considerada como información pública y de cuáles medios dispone el ciudadano para acceder a ella.

En síntesis de lo expuesto, los principales instrumentos jurídicos que regulan el Gobierno Digital en Costa Rica podrían representarse bajo el modelo de jerarquía de las normas, de la siguiente manera:
Gráfico 1. Fuente: creación propia con datos oficiales. Jerarquía de las normas del Gobierno Digital en Costa Rica.

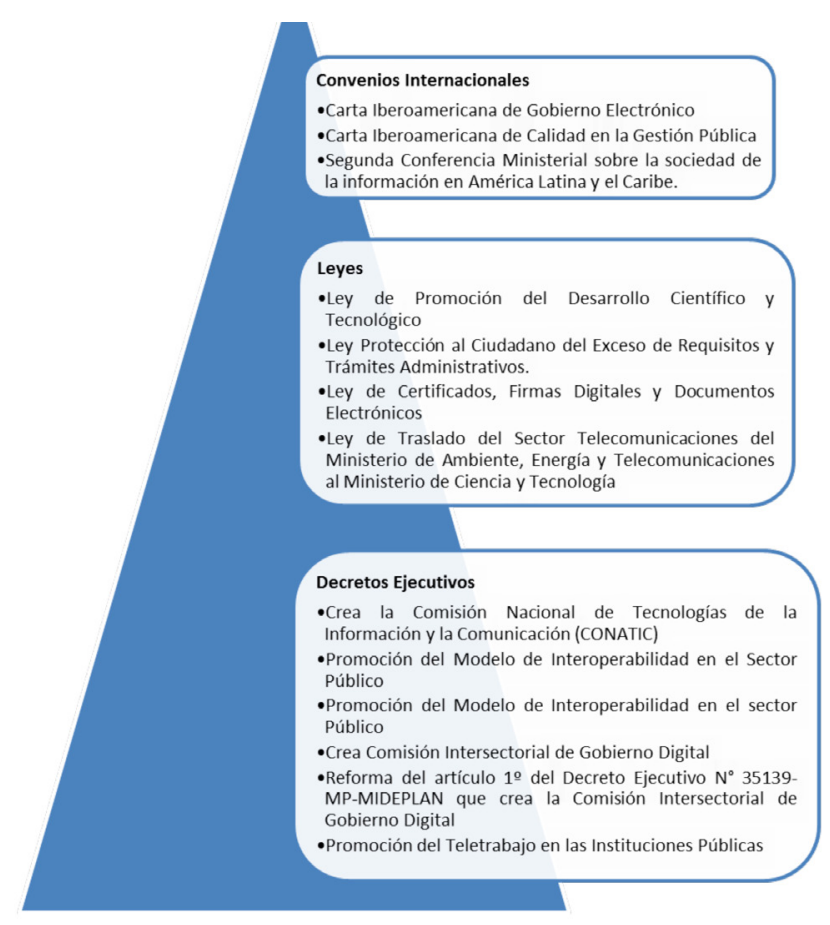

\section{PRINCIPALES ACTORES DEL GOBIERNO DIGITAL EN COSTA RICA}

\section{A. Secretaría Técnica del Gobierno Digital}

El primer Decreto Ejecutivo emitido con el objetivo de incursionar en el tema del Gobierno Digital fue el Decreto Ejecutivo $N^{\circ} 33147-M P$ del 8 de mayo del 2006, el cual ya no se encuentra vigente, pero que creó la: Comisión Intersectorial de Gobierno Digital, como un órgano de coordinación y definición política de alto nivel que diseña y planifica las políticas públicas en materia de Gobierno Digital. 
Igualmente, crea la Secretaría Técnica de Gobierno Digital como órgano adscrito a la Presidencia de la República e instrumento ejecutor de las políticas, estándares y proyectos que defina la Comisión, responsable de incrementar la eficiencia y la transparencia en el sector público a través del uso estratégico de las tecnologías digitales con el fin de empoderar a los habitantes en el uso de servicios.

Este decreto fue modificado por medio del Decreto Ejecutivo $N^{\circ} 34093$ denominado Reforma Comisión Intersectorial de Gobierno Digital, el cual modifica la composición de la comisión creada en el decreto anterior y delega en la Segunda Presidencia de la República la responsabilidad política de conducción de la Secretaría Técnica de Gobierno Digital.

En el 2008 se emite el Decreto Ejecutivo $N^{\circ}$ 34413 denominado: Reforma Integral del Decreto Ejecutivo $N^{\circ}$ 33147-MP que Crea la Comisión Intersectorial de Gobierno Digital y la Secretaría Técnica de Gobierno Digital, el cual reestructura la organización de la Secretaría Técnica de Gobierno Digital, tanto a nivel de las instituciones que conforman la Comisión Intersectorial como en la capacidad estratégica y ejecutiva de la Secretaría, mediante la incorporación de un asesor de dicha comisión y que viene a establecer una intermediación entre la dirección de la Secretaría y la comisión misma e, igualmente, traslada nuevamente la Secretaría a cargo de la Presidencia de la República.

En ese mismo año se emite el Decreto Ejecutivo $N^{\circ} 34702$ denominado: Se reforman parcial- mente los artículos $2^{\circ}$ y $4^{\circ}$ del decreto ejecutivo $N^{\circ} 33147$ que crea la comisión intersectorial de Gobierno Digital y la secretaría técnica de Gobierno Digital, el cual, nuevamente modifica la integración de la Comisión Intersectorial de Gobierno Digital.

Los anteriores decretos fueron derogados y entró en vigencia el Decreto Ejecutivo $N^{\circ}$ 35139-MPMIDEPLAN denominado, nuevamente: Crea Comisión Intersectorial de Gobierno Digital del 18 de marzo del 2009, en donde se traslada la Secretaría de Gobierno Digital al Instituto Costarricense de Electricidad (en adelante ICE), según el artículo $2^{\text {do }}$ que a la letra dice:

Artículo $2^{\circ}$-Con fundamento en la expresa disposición autónoma del ICE contenida en el "Convenio Marco de Cooperación Interinstitucional" entre el Gobierno de la República y el Grupo ICE suscrito el 5 de diciembre del 2006, designase al ICE como la Secretaría Técnica de Gobierno Digital.

La Secretaría Técnica de Gobierno Digital será el órgano ejecutor de las políticas, estándares y proyectos que defina la Comisión para incrementar la eficiencia y la transparencia en el Sector Público por medio del uso estratégico de las tecnologías digitales con el fin de empoderar a los habitantes en el uso de servicios públicos. Las políticas, estándares y proyectos serán comunicados a la CONATIC por medio del Ministerio de Ciencia y Tecnología.

EI ICE podrá determinar las vías de ejecución que considere convenientes para el cumplimiento de lo aquí dispuesto. Las ejecuciones podrán realizarse por su medio, a través de alguna de 
sus empresas o bien mediante alianzas estratégicas, con sujetos del Sector privado. Para ello, el ICE dispondrá de las facultades que dispone la Ley de Fortalecimiento y Modernización de las Instituciones Públicas del Sector Telecomunicaciones ( $N^{\circ} 8660$ del 8 de agosto del 2008).

El ICE nombrará un Gerente de la Secretaría Técnica de Gobierno Digital encargado de la ejecución de las políticas, estándares y proyectos que defina la Comisión. El Gerente asistirá a las reuniones de la Comisión. (Poder Ejecutivo, 2009).

Asimismo, los objetivos perseguidos con dicho traslado al ICE, se encuentran en el artículo $3^{\text {ro }}$ que dice:

Artículo $3^{\circ}$-EI ICE, conforme a la disposición autónoma descrita en el artículo 2, vigilará que se cumplan los siguientes objetivos:

A) Implementar herramientas digitales que faciliten el acceso a los servicios críticos para mejorar la calidad de vida de los habitantes, según el orden de prioridad que establezca la Comisión;

B) Incrementar la transparencia y acceso a la información gubernamental;

C) Facilitar los mecanismos de ciudadanía activa en su interacción con el Estado;

D) Fomentar el acceso a las tecnologías digitales entre los ciudadanos;

E) Promover la utilización de tecnologías digitales para la simplificación de trámites ante los órganos y entes públicos, así como las empresas del Estado y sus instituciones;
F) Incentivar la eficiencia del Estado y sus instituciones por medio del uso de las tecnologías digitales;

G) Velar por el modelo de Gobierno Digital que defina la Comisión y procurar su actualización;

H) Procurar el uso eficiente de los recursos gestionados por el Estado y sus instituciones para sustentar los proyectos de Gobierno Digital.

I) Coordinar con el Ministerio de Ciencia y Tecnología, en su calidad de Rector de las Tecnologías de Información, el cumplimiento de las políticas y los estándares establecidos por la Comisión para el Proyecto del Gobierno Digital.

J) Colaborar con la Comisión en las tareas que esta le asigne para el cumplimiento de sus objetivos;

K) Hacer de los procedimientos técnicos, administrativos y financieros de la Secretaría Técnica de Gobierno Digital, modelos de eficiencia que no sólo garanticen el buen funcionamiento de la Secretaría, sino que puedan servir de modelo a otras actividades de los costarricenses. (Poder Ejecutivo, 2009).

Por lo tanto, la Secretaría Técnica del Gobierno Digital en Costa Rica es actualmente una dependencia del Instituto Costarricense de Electricidad; no obstante, este hecho debe ser analizado con cuidado, primero, por la situación de competencia en que actualmente se encuentra el ICE en el tema de telecomunicaciones y, segundo, porque dicha Secretaría debe promover el Gobierno Digital en todo el país, por lo que se debe analizar su vinculación a una empresa pública en específico. 
Igualmente, se debe considerar que el ICE no posee potestades sobre las demás instituciones del sector público, lo que eventualmente podría considerarse como una debilidad para la Secretaría pues carece de potestad para imponer sus lineamientos al resto de sector público en virtud de que no existe una relación jerárquica entre el ICE y las demás instituciones del sector público.

\section{B. Comisión Intersectorial de Gobierno Digital}

Por su parte, el Decreto Ejecutivo 35139 denominado: Crea Comisión Intersectorial de Gobierno Digital, le impone a ella la labor de planificar y elaborar las políticas sobre Gobierno Digital, al respecto el artículo $1^{\text {ro }}$ dice: "Créase la Comisión Interinstitucional de Gobierno Digital, en adelante denominada 'la Comisión' como un órgano interinstitucional de coordinación y definición política para diseñar, planificar y elaborar las políticas públicas en materia de Gobierno Digital" (Poder Ejecutivo, 2009).

De esta manera, le corresponde a la Secretaría Técnica de Gobierno Digital implementar las políticas que diseñe esta Comisión, así lo señala el numeral 2 del citado decreto:

La Secretaría Técnica de Gobierno Digital será el órgano ejecutor de las políticas, estándares y proyectos que defina la Comisión para incrementar la eficiencia y la transparencia en el Sector Público por medio del uso estratégico de las tecnologías digitales con el fin de empoderar a los habitantes en el uso de servicios públicos. Las políticas, estándares y proyectos serán comunicados a la CONATIC por medio del Ministerio de Ciencia y Tecnología. (Poder Ejecutivo, 2009).

Por lo expuesto, esta Comisión define las políticas sobre las que la Secretaría tendrá la competencia para ejecutar, todo dentro del seno del ICE, institución que posee a su cargo la citada Secretaría.

\section{Ministerio de Ciencia y Tecnología}

La Ley $N^{\circ} 7169$ denominada Ley de Promoción Desarrollo Científico y Tecnológico y Creación del MICYT (Ministerio de Ciencia y Tecnología), se aprobó con el objetivo de promover el desarrollo científico y tecnológico en el país y la utilización de las tecnologías modernas; lo cual, incluye el tema del Gobierno Digital, aspecto que le fue adicionado en el inciso $\mathrm{K}$ del artículo $3^{\text {ro }}$, que textualmente dice: “k) Promover el desarrollo y uso de los servicios de telecomunicaciones dentro del marco de la sociedad de la información y el conocimiento, y como apoyo a sectores como salud, seguridad ciudadana, educación, cultura, comercio y gobierno electrónico" (Asamblea Legislativa, 1990. Negrilla mía).

Lo anterior es un reconocimiento expreso de la importancia del Gobierno Digital como parte del desarrollo científico y tecnológico del país, cuya rectoría corresponde a dicho ministerio, según el inciso g) del numeral 20 de la citada ley, que en lo que interesa señala: "g) Ejercer la rectoría del sector telecomunicaciones generando políticas públicas que permitan el cumplimiento de los objetivos enumerados en el artículo 2 de la 
Ley N. ${ }^{\circ} 8642$, Ley General de Telecomunicaciones" (Asamblea Legislativa, 1990).

Esta misma Ley crea, dentro del Ministerio de Ciencia y Tecnología, el Sistema Nacional de Ciencia Tecnología, que integra todos los actores del sector público y privado que participan y trabajan en la promoción del desarrollo de estos temas en el país, al respecto dicho numeral dice:

ARTICULO (SIC) 7.- Créase el Sistema Nacional de Ciencia y Tecnología, dentro del marco de sectorialización del Estado. El Sistema está constituido por el conjunto de las instituciones, las entidades y los órganos del sector público, del sector privado y de las instituciones de investigación y de educación superior, cuyas actividades principales se enmarquen en el campo de la ciencia y la tecnología, o que dediquen una porción de su presupuesto y recursos humanos a actividades científicas y tecnológicas. (Asamblea Legislativa, 1990, Num. 20).

Pese a lo anterior y dada la importancia que corresponde a la labor rectora que debe ejercer el Ministerio de Ciencia y Tecnología, genera preocupación que dicho ministerio no cuenta con los recursos suficientes para ejercer adecuadamente esta importante labor, situación que ha sido señalada por la propia Contraloría General de la República en el Informe DFOE-IFRIF-5-2012, cuando dice:

en relación con los demás ministerios, es uno de los que tiene más baja asignación presupuestaria a nivel del Poder Ejecutivo, a lo que se suma que, según el monto de su presupuesto devengado correspondiente a los años 2008 al 2011, un $62,62 \%$ se destina a diversas transferencias corrientes, las cuales tienen el objetivo de incentivar el desarrollo científico y tecnológico en el país. (Contraloría General de la República, 2013, p. 44).

De tal manera que si bien, el legislador le dio esta enorme tarea al Ministerio de Ciencia y Tecnología, no obstante, el Poder Ejecutivo no le transfiere los recursos suficientes para poder ejercerla adecuadamente.

Lo expuesto provoca que dicha rectoría resulte insuficiente y el avance que se haya logrado en la materia sea escaso y con muchos pendientes, por lo que resulta necesario que se revisen las obligaciones asignadas a dicho ministerio de frente a los recursos disponibles, con el fin de replantear esta función.

\section{Comisión de Tecnologías de la Informa- ción y la Comunicación (CONATIC)}

Mediante Decreto Ejecutivo $N^{\circ} 31681$ denominado: Crea la Comisión Nacional de Tecnologías de la Información y la Comunicación (CONATIC), se establece esta institución con el fin de proponer políticas en materia de Tecnologías de la Información y la Comunicación en el Sector público y coordinar proyectos en el área privada.

Textualmente, el numeral primero de dicho decreto dice:

Artículo $1^{\circ}-$ Créase la Comisión Nacional de Tecnologías de la Información y la Comunicación (CONATIC) adscrita al Ministerio de Ciencia y Tecnología, cuya misión será proponer las po- 
líticas y estrategias en materia de Tecnologías de la Información y la Comunicación dirigidas a las instituciones del Sector Público, así como recomendar lineamientos técnicos y administrativos que orienten el accionar en la materia. La CONATIC establecerá canales de coordinación con los sectores empresarial, académico y organizaciones no gubernamentales en proyectos y acciones del ámbito de las Tecnologías de la Información y la Comunicación, de interés manifiesto para dichos sectores, actuando a la vez como ente concertador entre las partes involucradas.

Para el funcionamiento de CONATIC se utilizarán los recursos tanto financieros como humanos ya existentes en el Ministerio de Ciencia y Tecnología y en las demás instituciones que la conforman. (Asamblea Legislativa, 2004).

Asimismo, dentro de los objetivos que persigue esta Comisión está precisamente el de promover el Gobierno Digital, al respecto el inciso f del artículo $2^{\text {do }}$ del citado texto legal, expresamente señala:

f. Promover la adopción de políticas que aseguren el mejor uso de los recursos informáticos de las entidades públicas, así como la integración de los sistemas de información del Sector Público, con miras al desarrollo del Gobierno Digital, que permitan un mejor, eficiente y oportuno servicio a los ciudadanos. (Asamblea Legislativa, 2004).

No obstante, pese a poseer un mandato tan claro y estar vigente el decreto que le dio origen y le establece dicha función, la Comisión no ha realizado acciones durante los últimos años para promover el Gobierno Digital en el país, dando como consecuencia que no se esté cumpliendo su función en este sentido.

De lo expuesto anteriormente se refleja que existe diversidad de actores participando en la promoción del Gobierno Digital que dependen de distintas entidades públicas, situación que gráficamente podemos apreciar de la siguiente manera:

\section{Gráfico 2. Fuente: creación propia con datos oficiales}

Principales actores administrativos del Gobierno Digital en Costa Rica.

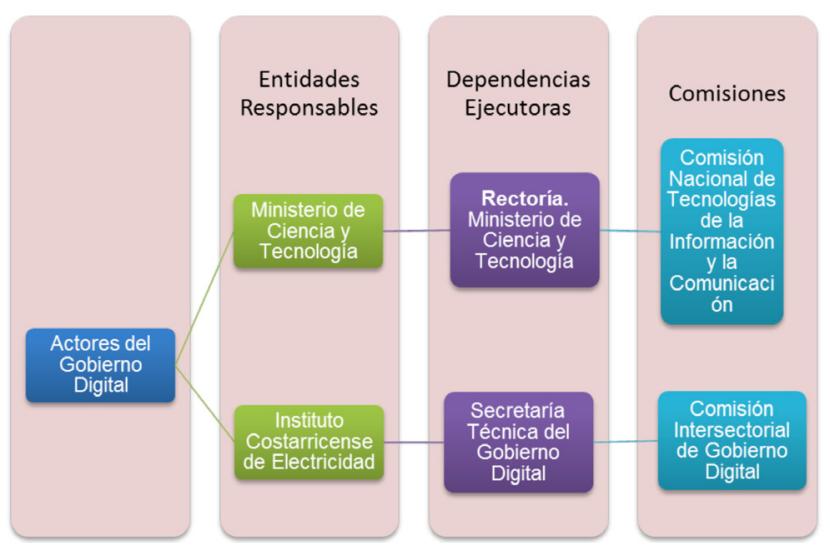

\section{PRINCIPALES OBSTÁCULOS PARA EL AVANCE DEL GOBIERNO DIGITAL EN COSTA RICA}

Pese a que se cuenta con normativa que pretende implementar e impulsar el Gobierno Digital en Costa Rica y que existen instituciones públicas a las cuales se les ha delegado esta labor, el avance del Gobierno Digital en dicho país no ha alcanzado el nivel óptimo y aún existe mucho trabajo pendiente en esta tarea, por lo que surge la pregunta: ¿cuáles han sido los obstáculos que han impedido el avance del Gobierno Digital 
en Costa Rica? ¿Qué aspectos se deben corregir para poder progresar en este tema?

En primera instancia se debe señalar que existen muchos actores participando en pro de este mismo objetivo, lo cual ha dificultado que se definan claramente las funciones, responsabilidades y competencias de cada uno; de tal manera que no se incurra en dualidad de funciones, o bien, en un conflicto de competencias.

Este pluralismo de actores ha conllevado a que no se pueda establecer políticas uniformes y aplicables para todo el sector público en la materia, dado que los distintos actores que participan en el proceso han definido sus propios mecanismos de trabajo. En otras palabras, se está ante una ausencia de articulación de políticas e instrumentos tendientes a impulsar el Gobierno Digital en este país.

Aunado a lo anterior, se debe mencionar que los actores antes mencionados no han realizado adecuadamente sus funciones, por ejemplo: el caso de la Comisión Nacional de Tecnologías de la Información y la Comunicación que no ha operado en los últimos años, unido a un actuar deficiente de los otros actores involucrados, conlleva a que sea poco el avance logrado en la materia.

Incluso, aquellos que han implementado acciones específicas carecen de instrumentos que garanticen que las acciones diseñadas sean sostenibles en el tiempo, es decir, que posean permanencia y continúen pese a los cambios políticos que se puedan dar en el gobierno.
Se carece de un verdadero liderazgo, pues si bien la normativa le otorga este papel al Ministerio de Ciencia y Tecnología, la realidad ha demostrado que carece de los recursos e instrumentos adecuados para realizar esta labor, lo cual, aunado a la ausencia de recursos presupuestarios para invertirlos en esta área, ya que este Ministerio no posee los fondos suficientes para destinarlos en proyectos de Tecnologías, que generalmente ascienden a grandes sumas de dinero, ha conllevado a que la labor desempeñada en esta materia resulte altamente deficiente.

Finalmente, se debe señalar que la ubicación de la Secretaria Técnica de Gobierno Digital dentro del Instituto Costarricense de Electricidad posee un alto riesgo de dualidad de mando al recibir políticas de la Comisión Intersectorial de Gobierno Digital e instrucciones del Consejo Directivo del ICE; y además, esta institución no posee competencia sobre el resto del sector público, lo que corresponde a una debilidad de la Secretaría para imponer sus acciones al sector público en general.

A manera de síntesis final, se puede esquematizar el modelo de Gobierno Digital en Costa Rica, de la siguiente manera: 
Gráfico 3. Fuente: creación propia con datos oficiales

Factores más relevantes del Gobierno Digital en Costa

Rica
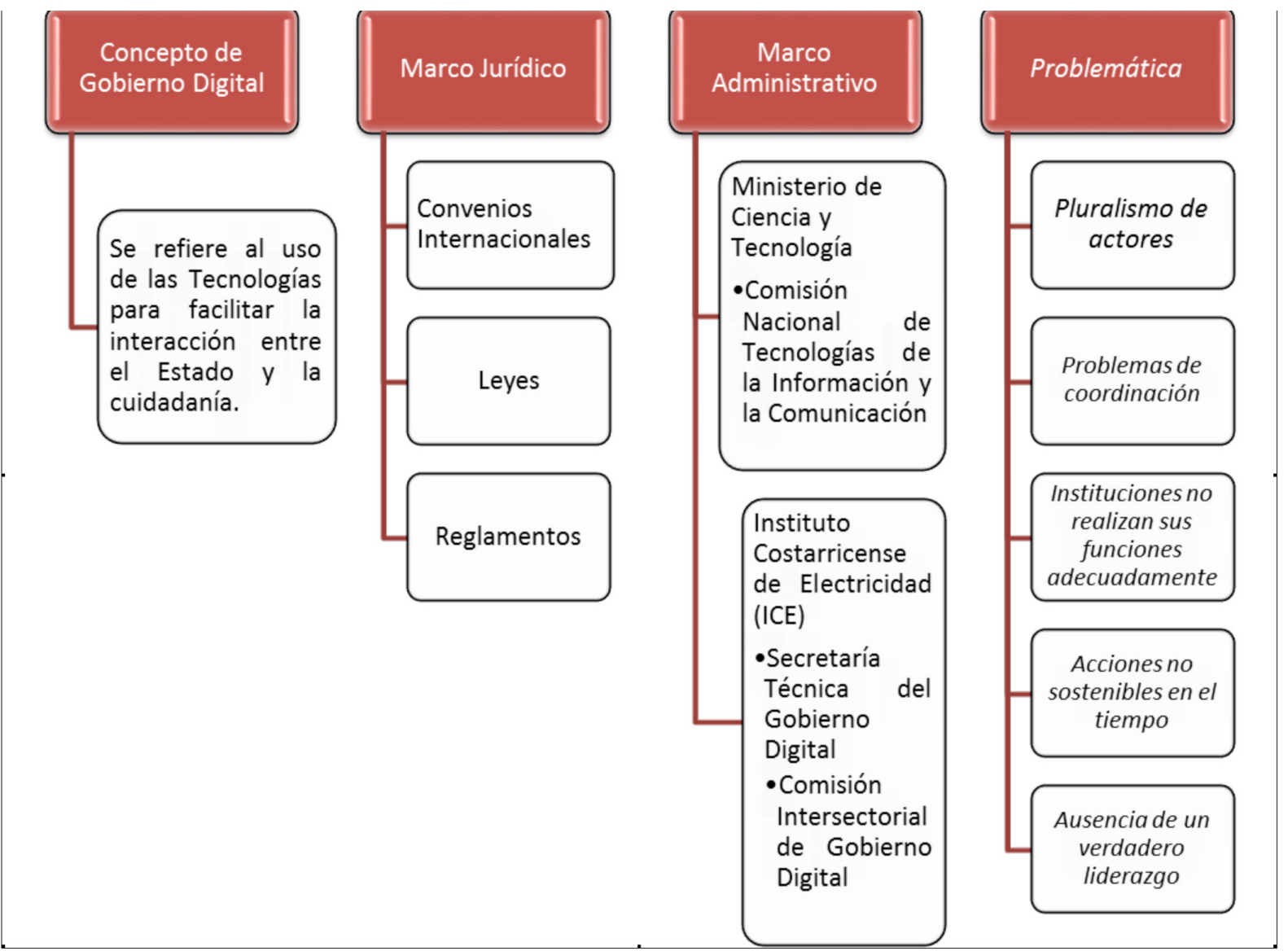

\section{PROPUESTA PARA SUPERAR LOS OBSTÁCULOS AL AVANCE DEL GO- BIERNO DIGITAL EN COSTA RICA}

En el apartado anterior se listaron los principales obstáculos que frenan el avance del Gobierno Digital en Costa Rica, por lo que el siguiente paso es preguntarse ¿qué se puede hacer ante esta situación?

Se debe empezar por señalar que el origen de la problemática se encuentra precisamente en ese pluralismo de actores y dualidad de mando, por lo que el Estado Costarricense debería cuestionarse sobre la pertinencia de mantener dos entidades responsables de los temas de Gobierno Digital y elegir a una de ellas para atribuirle la responsabilidad en esta materia.

Ahora bien, la atribución de responsabilidades debe venir, necesariamente, acompañada del otorgamiento de recursos, tanto económicos como humanos y jurídicos, para garantizar un avance en estos temas. Escasa efectividad posee un decreto que establezca una competencia 
a una institución, si ésta carece de los medios para ejecutarlo, tal y como actualmente acontece con el Ministerio de Ciencia y Tecnología, a quien se le asignó un papel importante en Gobierno Digital, pero su presupuesto no permite invertir en esta área, máxime cuando se toma en consideración que los proyectos de tecnologías requieren de altas inversiones económicas.

Por otra parte, junto con los recursos económicos se debe otorgar potestades legales para que las directrices que el ente rector emita tengan carácter vinculante para todo el sector público, de lo contrario, estas directrices pasarían a ser meras recomendaciones, tal y como ocurre actualmente con el ICE, que no tiene jerarquía sobre las demás instituciones del sector público $y$, por ende, no puede imponer sus criterios aun cuando formalmente posee un papel predominante en Gobierno Digital.

Recapitulando, resulta urgente que Costa Rica elimine esa dualidad de mando y elija a la entidad a la cual le va a otorgar la responsabilidad de dirigir el Gobierno Digital en el país y junto con ello le otorgue los recursos económicos, humanos, tecnológicos y jurídicos necesarios para que pueda desempeñar esa tarea.

Asimismo, esta entidad estaría obligada a asumir el liderazgo necesario para convertirse en el verdadero rector del sistema, que es uno de los problemas actuales más importantes, pues pese a que son muchas las instituciones involucradas, ninguna ha asumido ese rol de "rector" del tema.
Dentro de las primeras tareas que deberá asumir este rector, en ejercicio de sus funciones, es implementar adecuados mecanismos de coordinación con las entidades del sector público, tanto con aquellas que participan activamente de la creación del Gobierno Digital, como de las instituciones destinatarias de estos servicios y, del ciudadano como consumidor final.

En todo momento se debe tener presente que el objetivo principal del Gobierno Digital es mejorar los canales de comunicación entre el Estado y el ciudadano y que en última instancia estas acciones estén al servicio de la ciudadanía, por lo que el rector debe considerarlo como destinatario principal de sus políticas.

Finalmente, la problemática de permanencia en el tiempo de las acciones vinculadas con el Gobierno Digital debe abordarse integralmente, en primer lugar, el país debe reconocer la importancia de este tema y desligarlo de una promesa política, para evitar que cada vez que haya cambio de gobierno se modifiquen las acciones implementadas en la materia, por decirlo de alguna manera "despolitizarlo" y que pase a ser un aspecto técnico.

Y, en segundo lugar, los proyectos a implementar deben tener una visión de largo plazo y acciones permanentes en el tiempo que no se vean afectadas por cambios de gobierno, sino que continúen a pesar de los vaivenes políticos que afecten al país.

Para ello se retoma la necesidad de nombrar un rector en la materia, con suficiente capacidad técnica, recursos y poder jurídico para ejecutar 
proyectos de esta naturaleza, que requieren permanencia en el tiempo para que puedan dar resultados efectivos.

\section{CONCLUSIONES}

De lo anteriormente expuesto se puede concluir que Costa Rica ha realizado grandes esfuerzos por implementar el Gobierno Digital; ha dado signos positivos al adherirse a convenios internacionales en la materia y al promulgar normativa interna tendiente a armonizar el ordenamiento jurídico nacional con los estándares internacionales creando un ambiente propio para el crecimiento del Gobierno Digital en el país.

No obstante, estos esfuerzos no han alcanzado el desarrollo esperado, dado que dicha labor ha sido asignada a gran cantidad de instituciones, lo cual ha generado que exista un pluralismo de actores participando en el proceso con problemas de coordinación y armonización de los diferentes instrumentos que emiten, así como ausencia de un verdadero liderazgo en la materia, lo que ha conllevado a que se realicen esfuerzos aislados con poca permanencia en el tiempo.

De tal manera, el país requiere de manera urgente un replanteamiento en el tema, para lo cual se propone la definición de un ente encargado de la promoción de las estrategias tendientes a implementar el Gobierno Digital en Costa Rica con el fin de eliminar la dualidad de mando y los problemas de provocados por la pluralidad de actores.
Igualmente, se sugiere una mayor asignación de recursos al ente responsable de impulsar el Gobierno Digital, a fin de que posea las herramientas necesarias para poder cumplir con esta tarea; así como una revisión del marco jurídico que actualmente regula la materia con el objetivo de introducir las reformas necesarias para que este tema alcance el nivel de desarrollo que se desea y que el país merece, un Gobierno Digital eficiente a favor del ciudadano.

\section{Bibliografía}

XVII Cumbre Iberoamericana de Jefes de Estado y de Gobierno. (2007). Carta Iberoaméricana de Gobierno Electrónico. Chile: CLAD. Recuperada de http://www.bnp.gob.pe/ portalbnp/pdf/transparencia/normaslegales/2010/jun/carta_iberoamericana_de_gobierno_electronico.pdf

XVIII Cumbre Iberoamericana de Jefes de Estado y de Gobierno. (2008). Carta Iberoamericana de Calidad en la Gestión Pública. El Salvador: CLAD. Recuperado de http://www.clad.org/ documentos/declaraciones/carta-iberoamericana-de-calidad-en-la-gestion-publica/view

Asamblea Legislativa de la República de Costa Rica. (26 de junio de 1990). Ley N 7169 de 1990. Promoción del Desarrollo Científico y Tecnológico y Creación del MICYT (Ministerio de Ciencia y Tecnología). Recuperada de http://www.digeca.go.cr/documentos/legislacion/Ley\%20promocion\%20desarrollo\%20 cientifico\%20tecnologico.pdf 
--. (20 de enero de 2004). Decreto Ejecutivo $N^{\circ}$ 31681. Crea la Comisión Nacional de Tecnologías de la Información y la Comunicación (CONATIC). Recuperado de http://www.wipo. int/wipolex/es/text.jsp?file_id=222638

Banco Mundial. (s.f.). Concepto de Gobierno Digital. Recuperado de http://go.worldbank. org/M1JHEOZ280

Centro Latinoamericano de Administración para el Desarrollo. (2007). Carta Iberoamericana de Gobierno Electrónico. Aprobada por la IX Conferencia Iberoamericana de Ministros de Administración Pública y Reforma del Estado. Chile: Centro Latinoamericano de Administración para el Desarrollo. Recuperado de http://www.sgp.gov.ar/contenidos/cofefup/ documentos/docs/Carta_Iberoamericana_ de_Gobierno_Electronico.pdf

Comisión Económica para América Latina y el Caribe (CEPAL), Katz, J., Y Hilbert, M. (2003). Los caminos hacia una Sociedad de la Información en América Latina y el Caribe. Recuperado de http://www.eclac.cl/publicaciones/xml/5/11575/DGE2195-CONF91-3.pdf

Contraloría General de la República de Costa Rica. (2012). DFOE-IFR-IF-5-2012. Informe sobre las iniciativas que impulsan el desarrollo del Gobierno Digital y de una sociedad basada en la información y el conocimiento en Costa Rica. Recuperado de http://www.hacienda.go.cr/docs/51dc7283c011f_DFOEIFRIF52012-DGABCA.pdf
Fernando, G. (2004). Los Sistemas de Información en la Sociedad del Conocimiento. Madrid: ESIC Editorial.

Gobierno de El Salvador, Y Comisión Económica para América Latina y el Caribe (CEPAL). (2008). Segunda Conferencia Ministerial sobre la Sociedad de la Información de América Latina y el Caribe. Recuperado de http://www. eclac.org/cgi-bin/getProd.asp?xml=/socinfo/agenda/2/28042/P28042.xml\&base $=/$ socinfo/tpl/top-bottom.xsl

Organización de las Naciones Unidas, Y Unión Internacional de Telecomunicaciones. (2003). Declaración de Principios de la Cumbre Mundial de Sociedad de la Información. Construir la Sociedad de la Información: un desafío global para el nuevo milenio. Ginebra: Organización de las Naciones Unidas.

Organización de Estados Americanos. (2011). Módulo I: Introducción al Gobierno Electrónico. En Introducción a la Formulación de Estrategias de Gobierno Electrónico (pp. 1-25). Modalidad virtual: Organización de Estados Americanos.

Poder Ejecutivo de la República de Costa Rica. (8 de mayo de 2006). Decreto Ejecutivo $N^{\circ}$ 33147 de 2006. Crea la Comisión Intersectorial del Gobierno Digital. Recuperado de http://www.pgr.go.cr/scij/Busqueda/Normativa/Normas/nrm_repartidor.asp?param1= NRTC\&nValor1=1\&nValor2=57167\&param $2=1 \& \operatorname{strTipM}=T C \& \mid$ Resultado $=2 \& \operatorname{strSim}=s i$ $\mathrm{mp}$ 
---. (10 de octubre de 2007). Decreto Ejecutivo $N^{\circ}$ 34093. Reforma Comisión Intersectorial de Gobierno Digital. Recuperado de http://www. pgr.go.cr/scij/scripts/TextoCompleto.dII?Te xto\&nNorma $=61602 \& n$ Version $=76113 \& n T$ amanoLetra=10\&strWebNormativa=http:// www.pgr.go.cr/scij/\&strODBC $=D S N=S C I J$ $\mathrm{NRM} ; \mathrm{UID}=\mathrm{sa} ; \mathrm{PWD}=\mathrm{scij} ; \mathrm{DATABASE}=\mathrm{SCIJ}$ NRM;\&strServidor $=\backslash \backslash$ pgr04\&strUnidad=D:\& strJavaScript $=\mathrm{NO}$

---. (1 de abril de 2008). Decreto Ejecutivo $N^{\circ}$ 34413. Reforma Integral del Decreto Ejecutivo $N^{\circ}$ 33147-MP que Crea la Comisión Intersectorial de Gobierno Digital y la Secretaría Técnica de Gobierno Digital. Recuperado de http://www.pgr.go.cr/ scij/scripts/TextoCompleto.dll?Texto\&nNo $r m a=62791 \& n$ Version $=76114 \& n$ Tamano Letra $=10 \&$ strWebNormativa $=$ http://www. pgr.go.cr/scij/\&strODBC=DSN=SCIJ_NR $\mathrm{M} ; \mathrm{UID}=\mathrm{sa} ; \mathrm{PWD}=\mathrm{scij} ; \mathrm{DATABASE}=\mathrm{SCIJ}$ NRM;\&strServidor $=\backslash \backslash$ pgrO4\&strUnidad=D:\& strJavaScript $=\mathrm{NO}$

---. (6 de mayo de 2008). Decreto Ejecutivo $N^{\circ}$ 34702. Se reforman parcialmente los artículos $2^{\circ}$ y $4^{\circ}$ del decreto ejecutivo $N^{\circ} 33147$ que crea la comisión intersectorial de Gobierno Digital y la secretaría técnica de Gobierno Digital. Recuperado de http://www.pgr.go.cr/ scij//busqueda/normativa/Normas/nrm_articulo.asp?nValor1=1\&nValor $2=63804 \& n$ Valor $3=76116 \& n$ Valor $4=\&$ nValor $5=2 \& n$ Val or6 $=18 / 03 / 2009$

---. (6 de abril de 2009). Decreto Ejecutivo $N^{\circ}$ 35139-MP-MIDEPLAN. Crea Comisión Intersectorial de Gobierno Digital. Recuperado de http://www.pgr.go.cr/scij/Busqueda/Normativa/Normas/nrm_repartidor.asp?param1= NRTC\&nValor1=1\&nValor2=65185\&nValor3 $=82268 \&$ strTipM=TC

Sala Constitucional de la Corte Suprema de Justicia de la República de Costa Rica. (30 de Julio de 2010). Acceso a Internet como Derecho Fundamental, 12790-2010. Recuperado de https://docs.google.com/document/d/1_ n7anxwm9Cd4fJT-rP6zt1vvjHMnAODFibTVAMmCgO/edit

Secretaría Técnica de Gobierno Digital de Costa Rica. (s.f.). Gobierno Digital. Recuperado de http://www.gobiernofacil.go.cr/e-gob/gobiernodigital/index.html 